\title{
Measuring the effect of amantadine in chronic anoxic minimally conscious state
}

Caroline Schnakers, Roland Hustinx, Gilles Vanderwalle, Steve Majerus, Gustave Moonen, Melanie Boly, Audrey Vanhaudenhuyse, Marie-Aurélie Bruno, Steven Laureys

Introduction: The effect of pharmacological agents on recovery in chronic disorders of consciousness remains unsatisfactory. Amantadine, a dopaminergic agonist, has been suggested to behaviorally improve recovery from vegetative (VS) and minimally conscious states (MCS). Here, we report the effect of amantadine in a chronic anoxic MCS patient (i.e., presence of visual pursuit).

Methods: An ABAB design was proposed in conjunction with weekly CRS-R (Coma Recovery Scale-Revised) assessment, actimetry monitoring and serial (18F)fluorodeoxyglucose-PET (3 weeks baseline period, amantadine administrated during 6 weeks, stopped during 6 weeks and again resumed for 6 weeks).

Results: After 3 weeks of amantadine treatment, the patient showed reproducible movement to command and consistent automatic motor responses permitting mouth feeding. Similarly, actimetry monitoring showed a significant increase in limb movements during treatment periods. Finally, serial FDG-PET scanning objectively measured treatment-related metabolic changes in a widespread fronto-temporo-parietal network and in the sensori-motor area.

Conclusion: These data suggest a modulation of polymodal associative cortical metabolism and motor function by amantadine. Large scale studies on the efficiency of amantadine in this challenging patient population are warranted. 